\title{
Granulocytic sarcoma preceding leukaemic transformation in myelofibrosis
}

\author{
Peter Jacobs ${ }^{1,2}$ and Sean Sellars ${ }^{1,3}$ \\ ${ }^{1}$ The University of Cape Town Leukaemia Centre and the Departments of ${ }^{2}$ Haematology and ${ }^{3}$ Otolaryngology, \\ Groote Schuur Hospital, Observatory 7925, Cape, South Africa.
}

\begin{abstract}
Summary: A granulocytic sarcoma expanded the malar region in a patient with proven myelofibrosis over a 22 month period before undergoing rapid increase in size concomitantly with transformation to acute granulocytic leukaemia in the marrow and the widespread appearance of subcutaneous tumour deposits. Rapid response was obtained with local radiotherapy, and the systemic disease manifestations were controlled on combination courses of oral 4'-demethoxydaunorubicin and the epipodophyllotoxin VP16-213. This appears to be the first example of a granulocytic sarcoma occurring in a patient with myelofibrosis.
\end{abstract}

\section{Introduction}

Myeloblastoma appears to have been first described as a green tumour (Burns, 1823) and designated chloroma shortly thereafter (King, 1853). The association with acute leukaemia was originally misinterpreted (Dock, 1893) as lymphoid, and it was not until 1903 (Turk, 1903) that the correct correlation was made with the granulocytic cell line. The terminology for these tissue-destroying tumour nodules has changed. Chloroma, due to the green colour conferred upon them by the myeloperoxidase that they contained (Agner, 1941), is inappropriate since this is not a universal feature; myeloblastoma is also inexact, since the characteristic intracellular inclusions are not found in all patients. The preferred term is granulocytic sarcoma, based on the histological features of these neoplasms (Rappaport, 1966; Liu et al., 1973).

Granulocytic sarcomas have been reported to arise in many organs, characteristically as relatively late manifestations in the course of acute or chronic granulocytic leukaemia. This appears to be the first description in a patient with myelofibrosis only.

\section{Case report}

A 54 year old woman presenting in 1973 with anaemia, weight loss, and $1 \mathrm{~cm}$ splenomegaly, had a diagnosis of

Correspondence: Professor P. Jacobs, F.R.C.P.(Edin.), F.R.C.Path.(UK), Department of Haematology - Research Centre, University of Cape Town Medical School, Anzio Road, Observatory 7925, Cape, South Africa.

Accepted: 19 June 1985 myelofibrosis established on trephine biopsy. Five years later splenectomy was undertaken for increasing discomfort, pain due to infarction and increasing pancytopenia. Five years after the operation, whilst on titrated doses of hydroxyurea to control a rising granulocyte count, a non-tender firm swelling was noticed in the malar region of her face. Repeat trephine biopsy confirmed the presence in the bone marrow of myelofibrosis only. Radiological studies showed diffuse soft tissue swelling overlying the zygomatic process, with preservation of skeletal structures, including the sinuses. The tumour mass gradually increased in size over the next 18 months, became tethered to the underlying malar and maxilla but free of the overlying skin, and still without any radiological evidence of bone destruction.

At this time biopsy of enlarged groin glands showed granulocytic sarcoma, but marrow trephine biopsy again confirmed the presence of myelofibrosis only. The lymphadenopathy responded promptly to local radiotherapy. Haemoglobin was $8.1 \mathrm{~g} / \mathrm{dl}$, total white count $5.8 \times 10^{9} / 1$, with $30 \%$ neutrophils, $37 \%$ lymphocytes, $16 \%$ monocytes, $8 \%$ myeloblasts, $1 \%$ promyelocytes, $4 \%$ myelocytes, $4 \%$ metamyelocytes and platelets $100 \times 10^{9} / 1$; there were 164 nucleated red cells present for every 100 white cells counted.

Rapid swelling of the malar lesion now took place (Figure 1), and a sublabial biopsy showed the classical morphological features of a granulocytic sarcoma. This was treated with local radiotherapy and the face returned to normal within 4 weeks. Widespread discrete granulocytic sarcomas were now evident, and for the first time haematological evidence of 


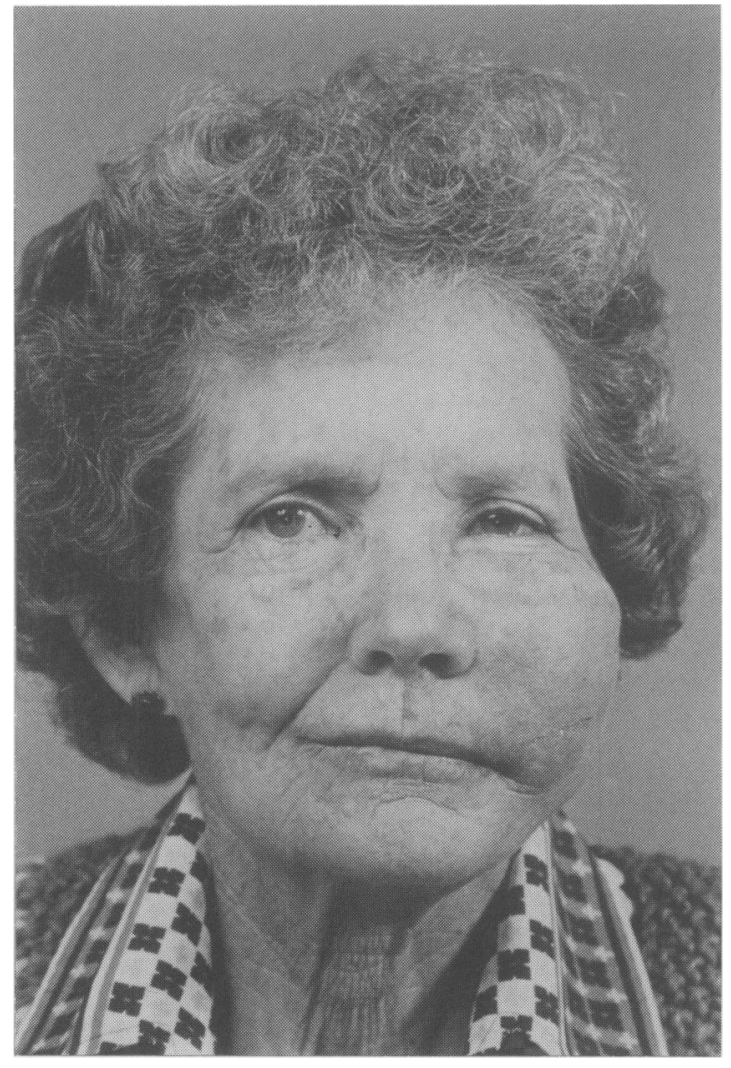

Figure 1 Extensive malar swelling narrowing the left palpebral fissure and distorting facial symmetry. After completion of radiotherapy all clinical and radiological evidence of the granulocytic sarcoma had disappeared.

myeloblastic transformation was seen, with $4 \%$ myeloblasts in the peripheral blood and extensive leukaemic involvement on trephine biopsy. Immediate resolution of all subcutaneous nodules was obtained with 4'-demethoxydaunorubicin and VP16-213. Reappearance of the granulocytic sarcomas, particularly in her legs, has been successfully controlled with intermittent courses of chemotherapy, without associated bone marrow suppression. The patient remains well and serial haematological reassessment shows peripheral blood and marrow regeneration without myeloblasts, and changes only of myelofibrosis persist.

\section{Discussion}

Granulocytic sarcomas appear not to have been previously described in the myeloproliferative syndrome, although their occurrence might be anticipated during the acute terminal phase of these syndromes, as has not infrequently been reported with myeloblastic transformation. In its classical form this tumour arises in the course of acute myelogenous or monoblastic leukaemia. Although it has been described in many different anatomical sites including small bowel (Brugo et al., 1975), breast mass during pregnancy (Sears \& Reid, 1976), cervix (Seo et al., 1977), uterus (Kapadia et al., 1978), ovary (Ballon et al., 1978), and perineural and epidural structures (Muss \& Moloney, 1973), and in the eye and the orbit (Cavdar et al., 1978), it does not appear to have been previously described as a soft tissue swelling in the malar region, where the present example is particularly remarkable for total preservation of skeletal structures in the area, including the sinuses. Furthermore, the presentation of a granulocytic sarcoma in patients with the myeloproliferative syndrome during the indolent phase of myelofibrosis, before clinical acceleration or myeloblastic transformation in the marrow has taken place, must be a unique event.

As a rule the tumours are radiosensitive and locah treatment to the groin glands promptly returned them to normal; the same gratifying response was seen in the patient's face. In general, myeloblastic transformation in the course of chronic granulocytic leukaemia responds poorly to therapy and survival is short. It is therefore worth noting that the oral regimen of 4' demethoxydaunorubicin in combination with the epipodophyllotoxin VP16-213 was dramatically effective and associated with only a transient period of marrow hypoplasia. Haematopoiesis regenerated without morphological evidence of acute leukaemia and although the period of follow-up is short, the convenience and efficacy of this regimen may warrant further evaluation.

\section{Acknowledgements}

Supported by the University of Cape Town Leukaemia Centre and Staff Research Fund, the Medical Research Council and the National Cancer Association. We thank Dr Andre Swanepoel for referring the patient, Professor David M. Dent for splenectomy, Dr Helen S. King for radiotherapy, Dr Bullet Myers for radiological studies, Jackie Davies for typing, and Dr H.- Reeve Sanders, Chief Medical Superintendent of Groote Schuur Hospital, for permission to publish. 


\section{References}

AGNER, K. (1941). Verdoperoxidase ferment isolated from leukocytes. Acta Physiologica Scandinavica, 2 (Suppl 8), 1.

BALLON, S.C., DONALDSON, R.C., BERMAN, M.L., SWANSON, G.A. \& BYRON, R.L. (1978). Myeloblastoma (granulocytic sarcoma) of the ovary. Archives of Pathology and Laboratory Medicine, 102, 474.

BRUGO, E.A., LARKIN, E., MOLINA-ESCOBAR, J. \& COSTANZI, J. (1975). Primary granulocytic sarcoma of the small bowel. Cancer, 35, 1333.

BURNS, A. (1823). In Observations on the Surgical Anatomy of the Head and Neck. American edition, p. 386. Baltimore: F. Lucas, Jr.

CAVDAR, A.O., ARCASOY, A., BABACAN, E. GAZDASOGLU, S., TOPUZ, U. \& FRAUMENI, J.F. (1978). Ocular granulocytic sarcoma (chloroma) with acute myelomonocytic leukemia in Turkish children. Cancer, 41, 1606.

DOCK, G. (1893). Chloroma and its relation to leukemia. American Journal of Medical Science, 106, 152.

KAPADIA, S.B., KRAUSE, J.R., KANBOUR, A.I. \& HARTSOCK, R.J. (1978). Granulocytic sarcoma of the uterus. Cancer, 41, 687.
KING, A. (1853). A case of chloroma. Monthly Journal of Medical Science, 17, 97.

LIU, P.I., ISHIMARU, T., MCGREGOR, D.H., OKADA, H. \& STEER, A. (1973). Autopsy study of granulocytic sarcoma (chloroma) in patients with myelogenous leukemia, Hiroshima-Nagasaki 1949-1969. Cancer, 31, 948.

MUSS, H.B. \& MOLONEY, W.C. (1973). Chloroma and other myeloblastic tumors. Blood, 42, 721.

RAPPAPORT, H. (1966). In Atlas of Tumor Pathology, section 3, fascicle 8, p. 241. Washington D.C.: Armed Forces Institute of Pathology.

SEARS, H.F. \& REID, J. (1976). Granulocytic sarcoma. Local presentation of a systemic disease. Cancer, 37, 1808.

SEO, I.S., HULL, M.T. \& PAK, H.Y. (1977). Granulocytic sarcoma of the cervix as a primary manifestation. Cancer, 40, 3030.

TURK, W. (1903). Akute myeloide leukaemie mit Gruenfaerbung der Knochenmarks miss. Gesellach. Fortschritte der Medizin, 2, 39. 